\title{
Mutations of the GLA gene in Korean patients with Fabry disease and frequency of the E66Q allele as a functional variant in Korean newborns
}

\author{
Beom Hee Lee ${ }^{1,2,9}$, Sun Hee Heo ${ }^{3,9}$, Gu-Hwan Kim ${ }^{2,3}$, Jung-Young Park ${ }^{3}$, Woo-Shik Kim ${ }^{4}$, Duk-Hee Kang ${ }^{5}$, \\ Kyung Hoon Choe ${ }^{6}$, Won-Ho Kim ${ }^{7}$, Song Hyun Yang ${ }^{8}$ and Han-Wook Yoo ${ }^{1,2,3}$
}

Fabry disease is caused by an $\alpha$-galactosidase A (GLA) deficiency. In this study, we identified 28 unrelated Korean families with Fabry disease with 25 distinct mutations in the GLA gene including six novel mutations (p.W47X, p.C90X, p.D61EfsX32, IVS4 ${ }^{-11} \mathrm{~T}>A$, p.D322E and p.W349). Notably, five subjects from four unrelated families carried the p.E66Q variant, previously known as a pathogenic mutation in atypical Fabry disease. Among these patients, only one had proteinuria and two had hypertrophic cardiomyopathy without any other systemic manifestation of Fabry disease. Substantial residual GLA activity was shown both in the leukocytes of p.E66Q patients (19.0-30.3\% of normal activity) and in transiently overexpressed COS-7 cells $(43.8 \pm 3.03 \%$ of normal activity). Although GLA harboring $\mathrm{p} . \mathrm{E} 66 \mathrm{Q}$ is unstable at neutral $\mathrm{pH}$, the enzyme is efficiently expressed in the lysosomes of COS-7 cells. The location of p.E66 is distant from both the active site and the dimer interface, and has a more accessible surface area than have other mutations of atypical Fabry disease. In addition, the allele frequency of p.E66Q determined in 833 unrelated Korean individuals was remarkably high at $1.046 \%$ (95\% confidence interval, $0.458-1.634 \%$ ). These results indicate that $\mathrm{p} . \mathrm{E} 66 \mathrm{Q}$ is a functional polymorphism rather than a pathogenic mutation. Journal of Human Genetics (2010) 55, 512-517; doi:10.1038/jhg.2010.58; published online 27 May 2010

Keywords: atypical variant; E66Q; Fabry disease; GLA; mutation; polymorphism

\section{INTRODUCTION}

Fabry disease (OMIM no. 301500) is an X-linked recessive lysosomal storage disorder caused by a deficiency of the $\alpha$-galactosidase A (GLA) enzyme (EC 3.2.1.22). ${ }^{1}$ GLA deficiency results in the accumulation of globotriaosylceramide (GL3), and related glycosphingolipids, in the lysosomes of various tissues and cell types. The incidence of Fabry disease was known to be 1 in 1250-117000 live male births worldwide. ${ }^{2-5}$

Clinical manifestations of Fabry disease are variable. Patients with classical Fabry disease present with acroparethesis, hypohidrosis, angiokeratoma, corneal opacities and varying degrees of autonomic dysfunction in childhood or adolescence, and suffer from chronic renal failure, hypertrophic cardiomyopathy or cerebral vascular events, which can lead to death in their second to fifth decade. ${ }^{3,6}$ Most male patients present with classical Fabry disease, whereas heterozygous females experience a wide spectrum of disease severity ranging from asymptomatic to presentation with classical disease. ${ }^{3,6}$
Patients with residual GLA activity show atypical Fabry disease with milder clinical manifestations. To date, three disease variants have been identified as atypical. The cardiac variant refers to manifestations confined to left ventricular hypertrophy, conduction abnormalities or cardiomyopathy without other systemic manifestations. ${ }^{7-10}$ Patients with the renal variant usually present with proteinuria and renal insufficiency without classical manifestations. However, most such patients also show cardiac manifestations, ${ }^{11-14}$ indicating that the renal variant might be intermediate between the cardiac variant and classical disease. More recently, the cerebral vascular variant was reported as causing acute strokes in young adults. ${ }^{15}$

Efforts have been made to explain the wide range of phenotypic heterogeneity in Fabry disease. To date, more than 500 mutations have been identified in the GLA gene, located at Xq22. The majority of mutations are private, restricted to only one or a few families (www.hgmd.cf.ac.uk/ac/index.php). More than $58 \%$ of mutations

\footnotetext{
${ }^{1}$ Department of Pediatrics, University of Ulsan College of Medicine, Asan Medical Center, Seoul, Korea; ${ }^{2}$ Medical Genetics Clinic and Laboratory, University of Ulsan College of Medicine, Asan Medical Center, Seoul, Korea; ${ }^{3}$ Genome Research Center for Birth Defects and Genetic Disorders, University of Ulsan College of Medicine, Asan Medical Center, Seoul, Korea; ${ }^{4}$ Division of Cardiology, Department of Internal Medicine, School of Medicine, Kyung Hee University, Seoul, Korea; ${ }^{5}$ Division of Nephrology, Department of Internal Medicine, Ewha Womans University School of Medicine, Ewha Medical Research Center, Seoul, Korea; ${ }^{6}$ Department of Cardiology, Wonju College of Medicine, Yonsei University, Wonju, Korea; ${ }^{7}$ Department of Cardiovascular Medicine, Medical School of Chonbuk National University, Jeonju, Korea and ${ }^{8}$ Institute of Metabolism, Green Cross Reference Laboratory, Seoul, Korea

${ }^{9}$ The authors contributed equally to this paper as the first author.

Correspondence: Dr H-W Yoo, Department of Pediatrics, Genome Research Center for Birth defects and Genetic Diseases, Asan Medical Center, University of Ulsan College of Medicine, 388-1 Pungnap-Dong, Songpa-Gu, Seoul 138-736, Korea.

E-mail: hwyoo@amc.seoul.kr
}

Received 24 February 2010; revised 20 April 2010; accepted 26 April 2010; published online 27 May 2010 
are missense or nonsense, whereas splicing, small or gross deletions or insertion mutations comprise the remainder. Most mutations in classical Fabry disease affect the catalytic activity of the enzyme, whereas mutations in atypical Fabry disease do not affect enzyme activity per se but rather lead to improper protein folding and, subsequently, instability of the tertiary structure. ${ }^{7,16-18}$ These mutant proteins retain some residual enzyme activity, which is related to the milder phenotypes of atypical Fabry disease.

In a previous report, we noted residual enzyme activities in COS-7 cells transiently overexpressing enzymes with the p.I91T and p.F113L mutations, which were identified in atypical Fabry disease. ${ }^{19}$ In this report, we found remarkably high residual enzyme activity of a protein with the p.E66Q mutation, previously known as a pathogenic mutation in patients with atypical Fabry disease. ${ }^{9,11,17,20}$ p.E66Q synthesizes $40 \%$ of wild-type enzyme activity when overexpressed in COS-7 cells, indicating that p.E66Q might be a mild mutation or a functional single nucleotide polymorphism (SNP). ${ }^{19}$

Herein, we report 25 distinct mutations in GLA, including six novel mutations, identified from 28 unrelated Korean families with Fabry disease. In addition, we review the clinical and biochemical findings of five patients with the p.E66Q variant and investigate the allele frequency of p.E66Q in the normal Korean population. We also discuss whether the p.E66Q variant should be classified as an SNP rather than a pathogenic mutation of Fabry disease.

\section{MATERIALS AND METHODS}

\section{Patients}

The institutional review boards of Asan Medical Center, Seoul, Korea, approved this study (no. 2009-0220). Each patient signed a written informed consent. All the subjects, including probands and their family members, were evaluated for Fabry disease based on its clinical features, markedly decreased activity of GLA and results of genetic tests in the GLA gene. From October 1998 to November 2009, 28 unrelated Korean families, comprised of 33 males and 16 females, had been diagnosed at the Asan Medical Center and enrolled in the Korean Fabry Registry. ${ }^{19,21,22}$ Clinical manifestations were reviewed in five patients from four families (family 25-28) with the p.E66Q variant. GL3 was measured both in plasma and urine of the patients. Plasma and urine were prepared with modification of the Bligh-Dyer method and evaluated by tandem mass spectrometry as described previously. ${ }^{23,24}$

\section{Molecular analysis of the GLA gene}

Genomic DNA was extracted from peripheral blood leukocytes using the Puregene DNA isolation kit (Gentra, Minneapolis, MN, USA). The PCR products covering 7 exons and their exon-intron boundaries were directly sequenced using the BigDye Terminator kit, version 3.1 (Applied Biosystems, Foster city,CA, USA). For the proband of family no. 22, total RNA was isolated from peripheral leukocytes using the Quickgene RNA kit (Fujifilm, TK, JP). cDNA was synthesized with Moloney-Monkey Leukemia Virus reverse transcriptase (Promega, Madison, WI, USA). Additional PCR and sequencing analysis of cDNA around exon 5 was performed to verify the aberrant splicing by the novel intronic variation, IVS4 $4^{-11} \mathrm{~T}>\mathrm{A}$.

\section{In vivo and in vitro biochemical assay of GLA activity}

In vivo GLA activity was determined in each patient's leukocytes using the fluorogenic substrate, 4-methylumbelliferyl-a-D-galactopyranoside (BioVectra, Oxford, CT, USA), as described earlier. ${ }^{25} \mathrm{~N}$-acetylgalactosamine $(0.1 \mathrm{M})$ (Amersham Pharmacia Biotech,Cedar Grove, NJ, USA) was used as an inhibitor of $\alpha$-Gal B activity. In vitro assay of GLA activity was conducted as described earlier. ${ }^{19}$

\section{DNA purification and TaqMan SNP genotyping assay}

A total of 833 Korean subjects born in 2008, 490 males and 343 females, were recruited. The genomic DNA of their neonatal dried blood spot was extracted
Table 1 Oligonucleotides used for detection of GLA fragment containing the p.E66Q site

\begin{tabular}{lll}
\hline Purpose & Oligonucleotides & Sequences $\left(5^{\prime} \rightarrow 3^{\prime}\right)$ \\
\hline Amplification & GLA exon2_fw & GGAGGTACCTAAGTGTTCATTTAATGAATTG \\
& GLA exon2_rev & AGCTCTGCCATCTCCATGAAG \\
RT detection & Probe wt-VIC & VIC-TCTTTCAGTGAGAAGCT-NFQ-MGB \\
& Probe mut-FAM & FAM-CTTTCAGTCAGAAGCT-NFQ-MGB \\
\hline
\end{tabular}

A reporter dye is linked to the $5^{\prime}$ end of each probe; VIC dye to the wild-type (p.E66) detection probe, and FAM dye to the mutant (p.E66Q) detection probe. The nucleotide complementary to the identified nucleotide variant is underlined.

from one or two dried blood spot disks, $3 \mathrm{~mm}$ in diameter, using the Generation Capture Card Kit (Qiagen, Hilden, Germany). The allele frequency of the p.E66Q variant in the Korean population was estimated using the TaqMan SNP genotyping assay. Real-time PCR was conducted for GLA SNP genotyping. $5^{\prime} \mathrm{VIC}$ and FAM probes, together with a $3^{\prime}$ non-fluorescent quencher (NFQ), were used as reporters of the p.E66-wild type and p.E66Q alleles, respectively. The sequences of wild type (p.E66) and variant (p.E66Q) probes are described in Table 1. Real-time PCR was carried out using the Chromo 4 Four-Color Real-Time System (MJ Research, Waltham, MA, USA). Fluorescent data generated by cleavage of the dual-labeled probes were collected during the PCR annealing step. Data analysis for allelic discrimination was conducted using the MJ Opticon Monitor Analysis software (version 3.1; MJ research). Neonatal dried blood spots with the p.E66Q variant allele were confirmed by GLA mutation analysis.

\section{Microsatellite analysis}

The DNA extracts were amplified by PCR using primers for microsatellite markers on chromosome X, namely; DXS1060, DXS987, DXS1226, DXS1214, DXS1068, DXS993, DXS1001, DXS1047 and DXS8091. Forward primers were labeled by fluorescent dye (Applied Biosystems). Genomic DNA amplification was generated with a GeneAmp PCR System 9700 (Applied Biosystems). Microsatellites were analyzed with an Applied Biosystems ABI 3130xL genetic analyzer.

\section{Immunofluorescence and confocal microscopy}

COS-7 cells were plated onto 35-mm 6-well culture plates without coverslips for immunofluorescence microscopy. The GLA expression vectors encoding wild type or mutant alleles were transfected into host cells using Lipofectamine 2000 (Invitrogen, Carlsbad, CA, USA) and incubated with rabbit anti-human GLA antibodies at $37^{\circ} \mathrm{C}$ for $48 \mathrm{~h}$ (Santa Cruz Biotechnology San Diego, CA, USA). The GLA antibody solution was removed, and cells were incubated with mouse anti-human LAMP1 antibody (Abcam, Cambridge, MA, USA) for lysosome identification or mouse anti-human PDI antibodies (Abcam) for endoplasmic reticulum (ER) identification. Subsequently, the cells were incubated with goat anti-rabbit IgG-RITC and goat anti-mouse-Rhodamine (Abcam). Slides were examined using a Leica TCS SP2 confocal laser scanning microscope (Leica Lasertechnik, Heidelberg, Germany).

\section{RESULTS}

Identification of 25 genotypes from 28 Korean families with Fabry disease

Twenty-five genotypes were identified in 28 Korean families with Fabry disease (Table 2). Twenty-two of the genotypes were associated with classical Fabry disease. Such patients had markedly decreased GLA activity in comparison with control levels, both in plasma and in transiently overexpressed COS-7 cells. Two other genotypes, p.I91T and p.F113L, were identified in renal variants, with residual GLA enzyme activity. Each family had a distinct mutation in the GLA gene. Six novel mutations, p.W47X, p.C90X, p.D61EfsX32, IVS4 ${ }^{-11} \mathrm{~T}>\mathrm{A}$, p.D322E and p.W349R, were identified. Decreased GLA activities were shown in the leukocytes of each patient and or in transfected COS-7 cells. PCR amplification and sequencing of cDNA around the region 
Table 2 The mutations and GLA activities in Fabry disease of Korean families

\begin{tabular}{|c|c|c|c|c|c|c|}
\hline \multirow[b]{2}{*}{ Family } & \multicolumn{2}{|c|}{ Nucleotide change } & \multirow[b]{2}{*}{ Location } & \multicolumn{2}{|c|}{ GLA activity (\% normal) } & \multirow[b]{2}{*}{ Phenotype } \\
\hline & Nucleotide & Amino acids & & $\begin{array}{c}\text { In vivo (percentage } \\
\text { mean normal) }\end{array}$ & $\begin{array}{c}\text { In vitro (percentage } \\
\text { wild-type) }\end{array}$ & \\
\hline 1 & c. $124 A>G$ & p.M42V & Exon 1 & 3.87 & $7.2 \pm 0.22$ & Classical \\
\hline 2 & c. $137 A>G$ & p.H46R & Exon 1 & 0.01 & NA & Classical \\
\hline 3 & c. $141 G>A^{c}$ & p.W47Xc & Exon 1 & 0.06 & NA & Classical \\
\hline 6 & c. $258 \mathrm{~T}>\mathrm{G}$ & p.Y86X & Exon 2 & 3.05 & $5.4 \pm 0.27$ & Classical \\
\hline 7 & c. $272 \mathrm{~T}>\mathrm{C}$ & p.191T & Exon 2 & 0.60 & $12.0 \pm 0.69$ & Renal \\
\hline 8 & c.296_297del & p.Q98fsX23 & Exon 2 & 0.00 & NA & Classical \\
\hline 9 & c. $334 \mathrm{C}>\mathrm{T}$ & p.R112C1 & Exon 2 & 0.00 & $4.7 \pm 0.13$ & Classical \\
\hline 10 & c. $337 \mathrm{~T}>\mathrm{C}$ & p.F113L & Exon 2 & 10.0 & $17.8 \pm 0.88$ & Renal \\
\hline 11 & c. $426 C>G$ & p.C142W & Exon 3 & 0.00 & $5.2 \pm 0.39$ & Classical \\
\hline 16 & c. $692 A>G$ & p.D231G & Exon 5 & 0.22 & $4.4 \pm 0.32$ & Classical \\
\hline 17 & c.803_806del & p.L268fsX1 & Exon 6 & 0.00 & NA & Classical \\
\hline 18 & $\mathrm{c} .890 \mathrm{C}>\mathrm{T}$ & p.S297F & Exon 6 & 0.21 & $5.9 \pm 0.45$ & Classical \\
\hline 19 & c. $902 \mathrm{G}>\mathrm{A}$ & p.R301Q & Exon 6 & 0.32 & NA & Classical \\
\hline 20 & c. $966 C>G^{c}$ & p.D322E $\mathrm{E}^{\mathrm{C}}$ & Exon 6 & 0.01 & NA & Classical \\
\hline 21 & c. $1024 \mathrm{C}>\mathrm{T}$ & p.R342X & Exon 7 & 0.00 & NA & Classical \\
\hline 22 & c. $1045 \mathrm{~T}>\mathrm{C}^{\mathrm{c}}$ & p.W349R & Exon 7 & 0.47 & $3.8 \pm 0.28$ & Classical \\
\hline 23 & c.1077del & p.I359MfsX32 & Exon 7 & 0.00 & NA & Classical \\
\hline 24 & c.1235_1236del & p.T412SfsX37 & Exon 7 & 0.00 & NA & Classical \\
\hline 25 & c. $198 \mathrm{G}>\mathrm{A}$ & p.E66Q & Exon 2 & 19.0 & $43.8 \pm 3.03$ & ND \\
\hline 26 & c. $198 \mathrm{G}>\mathrm{A}$ & p.E66Q & Exon 2 & NA & $43.8 \pm 3.03$ & ND \\
\hline 27 & c. $198 \mathrm{G}>\mathrm{A}$ & p.E66Q & Exon 2 & 36.3 & $43.8 \pm 3.03$ & ND \\
\hline
\end{tabular}

Abbreviations: NA, not-available; ND, not-determined.

a Normal range of GLA activity in leukocytes was $72.41 \pm 34.66 \mathrm{nmol} / \mathrm{hr} / \mathrm{mg}(n=99) .{ }^{19}$

${ }^{\mathrm{b} A c t i v i t i e s}$ of GLA were measured in vitro by overexpression in COS-7 cells. ${ }^{19}$

cNovel mutation.

of exon 5 in the proband of family 12 with IVS $4^{-11} \mathrm{~T}>\mathrm{A}$ showed the skipping of exon 5 , indicating the aberrant splicing by the mutation.

\section{Clinical, biochemical and molecular biological characteristics of the p.E66Q variant}

Four families (families 25-28, patients 1-5) harbored the p.E66Q variant (Table 2). Patient 1 developed chronic diarrhea with cramping epigastric pain, and a sense of tingling in the extremities at 2 years of age (Table 3). A gastroscopic examination showed reflux esophagitis with chronic gastritis. However, a nerve conduction study and electroencephalography yielded normal findings. Hypohidrosis, angiokeratoma, proteinuria, renal insufficiency and cardiac involvement were also absent. GLA activity in leukocytes assessed to rule out Fabry disease was decreased to $19.0 \%$ of the normal mean value $\left(72.47 \mathrm{nmol}^{-1} \mathrm{~h}^{-1} \mathrm{mg}^{-1}\right)$ (Table 2). A kidney biopsy revealed minimal mesangial hypercellularity without tubulointerstitial change and no myeloid body was noted. GL3 levels were normal in both plasma and urine. Enzyme replacement therapy (ERT), initiated at 2.5 years of age was, however, discontinued after 1 year because of diagnostic uncertainty, as well as no clinical improvement. Indeed, the patient did not show any manifestation of Fabry disease until 8 years of age. The abdominal pain and diarrhea improved with the administration of antacids. Patient 2, the mother of patient 1 and a carrier of p.E66Q, has shown no clinical signs of Fabry disease to her current age of 45 years. Patients 3 and 4 were evaluated for Fabry disease because of left ventricular hypertrophy of unknown origin. However, it neither showed proteinuria nor renal failure. Patient 5 was examined because of proteinuria $\left(8 \mathrm{~g} \mathrm{day}^{-1}\right)$. A renal biopsy revealed foamy or vacuolar cytoplasmic changes in glomerular epithelial cells, mesangial cells, endothelial cells, the smooth muscle of blood vessels and some tubular cells. No myeloid body was noted. She had no cardiac manifestation. Plasma and urine GL3 were normal. Proteinuria decreased to $1 \mathrm{~g} \mathrm{day}^{-1}$ in 4 months with a small dose of angiotensin II receptor antagonist (Losartan, $25 \mathrm{mg} \mathrm{day}^{-1}$ ). Patients 3-5 did not suffer angiokeratoma, acroparesthesia, hypohidrosis or hearing loss. Currently, none of these five patients received ERT (Table 3).

The residual enzyme activity in each patient's leukocytes was 19.0$30.3 \%$ of mean normal activity (normal range, $72.41 \pm 34.66 \mathrm{nmol}^{-1}$ $\mathrm{h}^{-1} \mathrm{mg}^{-1}$ ). Moreover, the enzyme activity of p.E66Q variant was $43.8 \pm 3.03 \%$ of normal activity in transiently overexpressed COS-7 cells (Table 2). Confocal microscopy showed that the p.E66Q variant GLA was expressed in lysosomes, a pattern similar to that of wild-type GLA, whereas the enzyme with the p.R112C mutation, a pathogenic mutation in atypical Fabry disease and that retains $4.7 \pm 0.13 \%$ of normal activity, ${ }^{19}$ was not expressed in lysosomes, but was rather retained in the endoplasmic reticulum (ER) (Figure 1). 
Table 3 Clinical findings of the five subjects with the p.E66Q variant

\begin{tabular}{|c|c|c|c|c|c|c|c|c|c|c|c|c|c|}
\hline \multirow[b]{3}{*}{ Family } & \multirow[b]{3}{*}{ Patient } & \multirow{2}{*}{\multicolumn{2}{|c|}{$\begin{array}{c}\text { Age at } \\
\text { detection }\end{array}$}} & \multirow[b]{3}{*}{ Paresthesia } & \multirow[b]{3}{*}{ Anhydrosis } & \multirow[b]{3}{*}{ Angiokeratoma } & \multirow{3}{*}{ Gastrointestinal } & \multirow[b]{3}{*}{ Heart } & \multirow[b]{3}{*}{ Kidney } & \multirow[b]{3}{*}{$S N H L$} & \multicolumn{2}{|r|}{ GL3 } & \multirow[b]{3}{*}{$E R T$} \\
\hline & & & & & & & & & & & Plasma & Urine & \\
\hline & & Sex & (years) & & & & & & & & $(3.88-9.87 \mu \mathrm{g} / \mathrm{ml})$ & $(0.01-0.90 \mu \mathrm{g} / \mathrm{mg} \mathrm{Cr})$ & \\
\hline 25 & Patient 1 & $M$ & 2 & $( \pm)$ & $(-)$ & $(-)$ & ND & $(-)$ & $(-)$ & $(-)$ & 4.282 & 0.079 & $(+)^{\mathrm{a}} \rightarrow(-)$ \\
\hline 25 & Patient 2 & $\mathrm{~F}$ & 41 & $(-)$ & $(-)$ & $(-)$ & $(-)$ & $(-)$ & $(-)$ & $(-)$ & NA & NA & $(-)$ \\
\hline 26 & Patient 3 & $M$ & 53 & $(-)$ & $(-)$ & $(-)$ & $(-)$ & $(+)$ & $(-)$ & $(-)$ & NA & NA & $(-)$ \\
\hline
\end{tabular}

Abbreviations: SNHL, sensorineuronal hearing loss; GL3, globotriaosylceramide; ND, not-determined; NA, not-available.

aDiscontinued after 1 year of ERT due to diagnostic uncertainty.

a

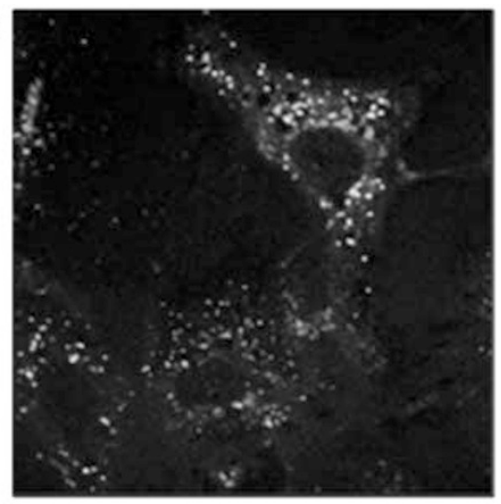

c

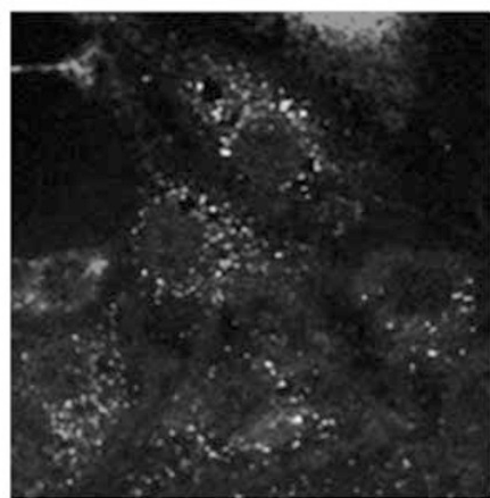

b

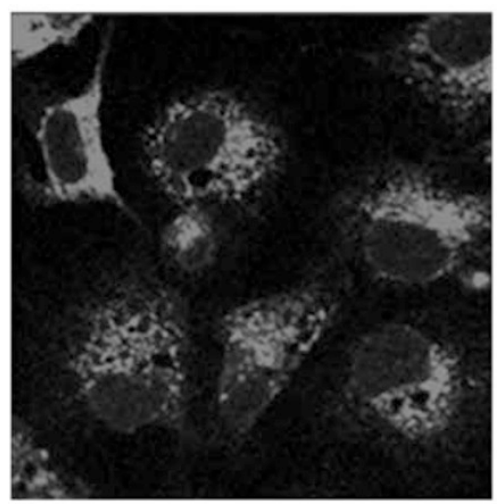

d

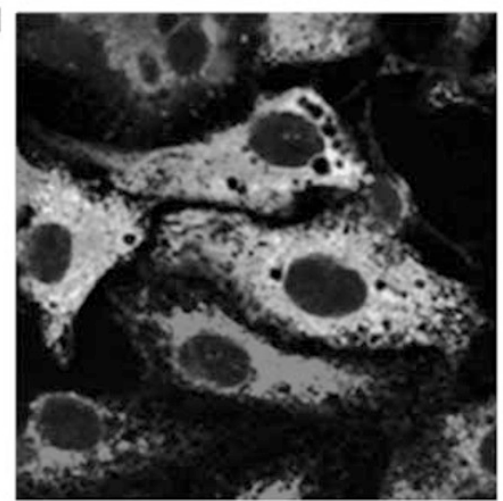

Figure 1 Confocal microscopy showing subcellular localization of expressed mutant constructs. Transfected COS-7 cells were double-stained with both rabbit anti-human a-Gal A antibodies and either mouse anti-human LAMP1 antibodies (a, c), as the lysosomal marker, or mouse anti-human PDI antibodies (c, d), as the ER marker. Panels: a, pWT (wild-type); b, mock transfection; c, p.E66Q; and d, p.R112C.

Allele frequencies of the p.E66Q variant in the Korean population Among 1176 alleles tested, 12 were found to harbor the p.E66Q variant. Two alleles were hemizygotes from two males and the remaining 10 heterozygotes from 10 females. The allele frequency in the Korean population was thus determined to be $1.046 \%$ (95\% confidence interval, $0.458-1.634 \%)$. Analyses of microsatellite markers revealed that none of the 833 subjects was related.

\section{DISCUSSION}

In this report on Korean Fabry families, all mutations in the GLA gene, excluding p.E66Q (24/25), were private, in that they were restricted to an individual family, and widely distributed along the gene, as in other populations. ${ }^{26}$ The characteristics of the mutations were also similar to those in other populations. Most mutations were point mutations (83.4\%), including missense (50.0\%), nonsense (29.2\%), and splicing (4.2\%) mutations, although a few deletion or insertion mutations were found to cause frame-shifts $(16.6 \%){ }^{26}$

With the advent of ERT, Fabry disease has become a treatable lysosomal storage disease. ${ }^{3,6}$ However, ERT has been most effective when initiated in the early stages of disease, and the long-term efficacy of ERT in the later stage of disease for prevention of complications such as renal failure, cardiac failure or stroke, has not yet been determined. ${ }^{6,15}$ To date, $0.25-1 \%$ of males receiving dialysis therapy because of renal failure, $3-4 \%$ of males with hypertropic cardiomyopathy, and $5 \%$ of males with acute cryptogenic stroke, have been identified as having Fabry disease. ${ }^{2,5,10-13,15,27}$ In this respect, a screening 
program to identify patients at early stages of disease is of course important for optimal patient prognosis. Moreover, recent two neonatal screening tests revealed that Fabry disease is more prevalent than had been expected, with an incidence of 1 in 1250-4600 individuals. ${ }^{4,5}$ However, these results require careful interpretation because most patients identified in the neonatal screening program carry mutations that are known to cause atypical Fabry disease, or mutations that were previously unknown. Because Fabry disease is a life-long condition that may lead to devastating complications during both early and late adulthood, genetic counseling of patients and their families identified by neonatal screening is a very difficult issue, especially from the ethical view point.

Although the p.E66Q variant used to be considered a pathogenic mutation, the remarkably high frequency of this allele in the Korean population $(>1 \%)$ and the substantial residual enzymatic activity indicate that a careful and comprehensive evaluation is warranted to assess the role of p.E66Q in the development of Fabry disease.

With the exception of one report in which p.E66Q was one of the compound heterozygotes found in patients with classical Fabry disease, p.E66Q has been identified in only renal or cardiac variants of Fabry disease. ${ }^{9,11,17,20}$ However, the clinical manifestations of patients with p.E66Q in the cited reports were not sufficiently convincing to confirm p.E66Q as a disease-causing mutation. For instance, Nakao et al. ${ }^{11}$ suggested that renal variants have a phenotype intermediate between cardiac variants and classical phenotypes. However, in contrast to their suggestion, the patient with p.E66Q had no cardiac manifestation although he was suffering from end-stage renal disease. The renal biopsy result was not available and the elevation of plasma GL3 was unremarkable. Furthermore, as reported by Yoshitama et al. ${ }^{9}$, a proband with p.E66Q showed asymmetric septal hypertrophy, which can make diagnosis of cardiomyopathy by Fabry disease indistinguishable from cardiomyopathy of other causes. Moreover, residual GLA activity was high, at $28.5-32 \%$ of the normal level in plasma and $28 \%$ of normal in leukocytes. ${ }^{9}{ }^{11}$ Similarly, the clinical characteristics of the patients described in our report were not sufficiently convincing to permit diagnosis of atypical Fabry disease. Although two patients (patients 3 and 4) with p.E66Q had cardiomyopathy, no patient showed any other manifestations. Widespread vacuolar changes in mesangial and endothelial cells in the renal biopsy of patient 5 are known as rare findings in Fabry disease. ${ }^{28}$ Normal GL3 levels and rapid decrease of proteinuria with small dose of anti-proteinuric medication also hindered the diagnosis. In addition, patient 5 did not show any cardiac manifestation. Although Fabry disease was suspected in patient 1 , the onset was much earlier than has been reported in pediatric Fabry disease patients..$^{29,30}$ In addition, the build up of GL-3 has not been observed. The pain in the extremities was not neuronopathic and the renal biopsy showed no findings suggestive of Fabry disease. Furthermore, relief from pain in the abdomen and extremities was improved by the administration of antacid medications, and not by ERT.

In contrast to other genotypes causing atypical Fabry disease with residual activities of $1.9-11.9 \%$ of normal enzyme activity, the p.E66Q enzyme retained a substantially higher activity (29-31\% of mean normal activity) in plasma or leukocytes and $30 \%$ of mean normal activity in transiently over-expressed COS-7 cells. ${ }^{8,17,19,20}$ Indeed, we have reported p.E66Q activities as high as $40 \%$ of mean normal activity in the transiently overexpressed COS-7 cells. ${ }^{19}$

$\mathrm{X}$-ray crystallography shows that GLA is a homodimeric glycoprotein in which each monomer is composed of two domains (Figure 2). The $N$-terminal domain 1 contains the active site in a $(\beta / \alpha)_{8}$ barrel, whereas the C-terminal domain 2 contains eight anti-parallel $\beta$ sheets. Most mutations in atypical Fabry disease are not associated with the catalytic site, but are rather buried in the interior of GLA, causing improper enzyme folding and subsequent degradation in the ER. The protein is predicted to show more residual enzyme activity when a mutation occurs in a more surface-accessible residue. ${ }^{17,31}$ The location of p.E66 is distant from both the active site and the dimer interface, and has a more accessible surface area of $3.1 \AA^{2}$ than have other mutations of atypical Fabry disease (Figure 2). ${ }^{31,32}$ Although predicted to be unstable at neutral $\mathrm{pH},{ }^{17}$ which is similar to the $\mathrm{pH}$ of the ER,

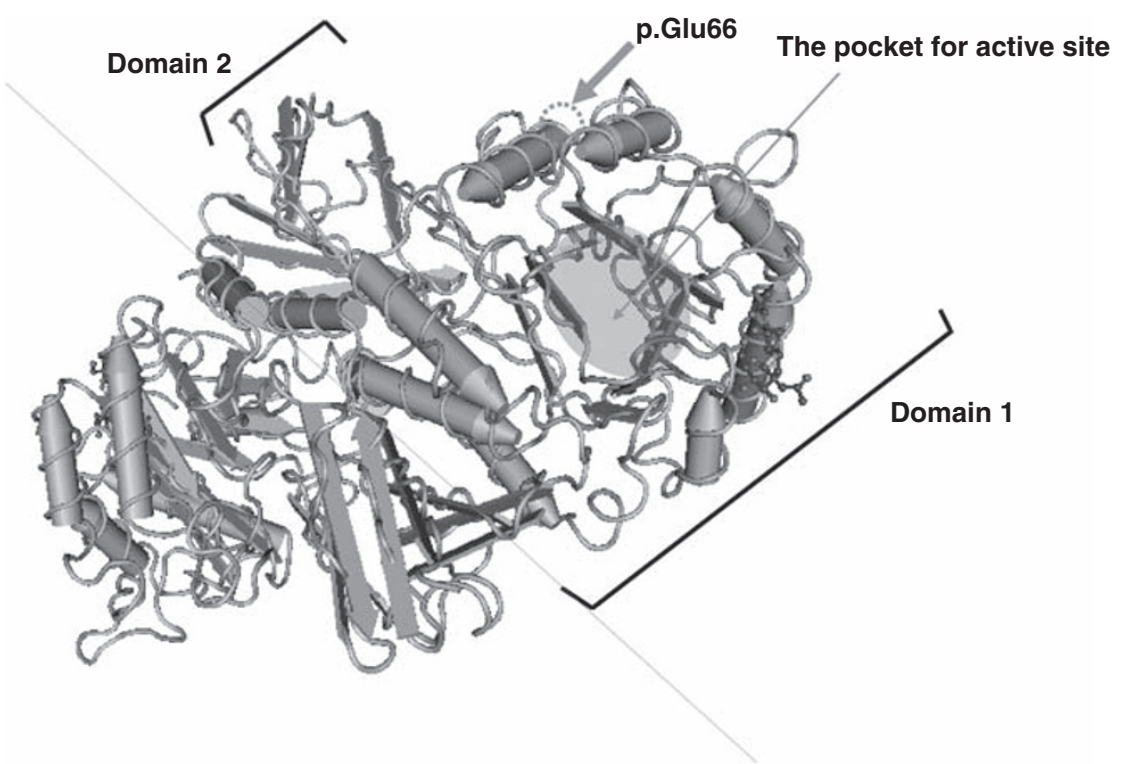

Figure 2 3D structure of GLA. Domain 1 in the $N$ terminus contains the active site in ( $\beta / \alpha)_{8}$ barrels (green color), whereas domain 2 in the $C$ terminus contains 8 antiparallel $\beta$ sheets (brown color). p.E66 (yellow color) is distant from both the active site and dimer interface, and bears more accessible surface area of $3.1 \AA^{2}$ (Garman, 2007). MMDB ID78.07 for GLA was presented using Cn3D, ver.4.1, available at the US National Center of Biotechnology Information, http://www.ncbi.nlm.nih.gov/. 
our confocal microcopy results showed that the p.E66Q enzyme is more easily localized in the lysosomes (where GLA is more stable) than seen in other genotypes of atypical Fabry disease. Similar findings were noted for another variant, p.D313Y, which was previously classified as a disease-causing mutation but is now considered to be a functional SNP. Although the p.D313Y variant was unstable at neutral $\mathrm{pH}$, the enzyme showed up to $60 \%$ of mean normal activity in transiently overexpressed cells, and was localized in lysosomes. ${ }^{33}$

Considering the genotypic and phenotypic heterogeneities found in Fabry disease, environmental factors or modifying genes may affect phenotypic severity among patients with p.E66Q. ${ }^{11,34}$ In addition, because underlying diseases of the five patients in the current report are still undetermined, isolated proteinuria or hypertrophic cardiomyopathy can be the only manifestation in atypical Fabry disease, the age of patient 1 at the latest evaluation was only eight, and patient 3 and 5 are heterozygous carriers of p.E66Q, atypical Fabry disease might not be ruled out confidently in these patients. Therefore, longterm follow-up of these patients with p.E66Q are necessary for close observation of disease progression in the absence of ERT by GL-3 monitoring in urine and plasma.

In conclusion, this is the first report on a large Korean cohort with Fabry disease, revealing genotypic heterogeneities in the GLA gene. The p.E66Q previously classified as a disease-causing mutation in atypical Fabry disease encodes $40 \%$ of wild-type enzymatic activity in cells, and the enzyme readily accesses the lysosome. In addition, the high frequency $(1.046 \%)$ of the allele in the Korean population and the lack of clinical features suggestive of Fabry disease, indicate that p.E66Q is not a disease-causing mutation but rather a functional SNP.

\section{CONFLICT OF INTEREST}

The authors declare no conflict of interest.

\section{ACKNOWLEDGEMENTS}

We thank the patients and their families for participating in this study, which was supported by a grant from the Korean Ministry for Health, Welfare and Family Affairs (Grant no. A080588).

1 Kornreich, R., Desnick, R. J. \& Bishop, D. F. Nucleotide sequence of the human alphagalactosidase A gene. Nucleic Acids Res. 17, 3301-3302 (1989).

2 Meikle, P. J., Hopwood, J. J., Clague, A. E. \& Carey, W. F. Prevalence of lysosomal storage disorders. JAMA 281, 249-254 (1999).

3 Zarate, Y. A. \& Hopkin, R. J. Fabry's disease. Lancet 372, 1427-1435 (2008)

4 Hwu, W. L., Chien, Y. H., Lee, N. C., Chiang, S. C., Dobrovolny, R., Huang, A. C. et al. Newborn screening for Fabry disease in Taiwan reveals a high incidence of the lateronset GLA mutation c.936+919G > A (IVS4+919G > A). Hum. Mutat. 30, 1397-1405 (2009).

5 Spada, M., Pagliardini, S., Yasuda, M., Tukel, T., Thiagarajan, G., Sakuraba, H. et al. High incidence of later-onset fabry disease revealed by newborn screening. Am. J. Hum. Genet. 79, 31-40 (2006)

6 Eng, C. M., Germain, D. P., Banikazemi, M., Warnock, D. G., Wanner, C., Hopkin, R. J. et al. Fabry disease: guidelines for the evaluation and management of multi-organ system involvement. Genet. Med. 8, 539-548 (2006).

7 Ishii, S., Kase, R., Sakuraba, H. \& Suzuki, Y. Characterization of a mutant alphagalactosidase gene product for the late-onset cardiac form of Fabry disease. Biochem. Biophys. Res. Commun. 197, 1585-1589 (1993).

8 Nakao, S., Takenaka, T., Maeda, M., Kodama, C., Tanaka, A., Tahara, M. et al. An atypical variant of Fabry's disease in men with left ventricular hypertrophy. $N$ Engl. J. Med. 333, 288-293 (1995).
9 Yoshitama, T., Nakao, S., Takenaka, T., Teraguchi, H., Sasaki, T., Kodama, C. et al. Molecular genetic, biochemical, and clinical studies in three families with cardiac Fabry's disease. Am. J. Cardiol. 87, 71-75 (2001).

10 Monserrat, L., Gimeno-Blanes, J. R., Marin, F., Hermida-Prieto, M., Garcia-Honrubia, A., Perez, I. et al. Prevalence of fabry disease in a cohort of 508 unrelated patients with hypertrophic cardiomyopathy. J. Am. Coll. Cardiol. 50, 2399-2403 (2007).

11 Nakao, S., Kodama, C., Takenaka, T., Tanaka, A., Yasumoto, Y., Yoshida, A. et al. Fabry disease: detection of undiagnosed hemodialysis patients and identification of a 'renal variant' phenotype. Kidney Int. 64, 801-807 (2003).

12 Kotanko, P., Kramar, R., Devrnja, D., Paschke, E., Voigtlander, T., Auinger, M. et al. Results of a nationwide screening for Anderson-Fabry disease among dialysis patients. J. Am. Soc. Nephrol. 15, 1323-1329 (2004).

13 Tanaka, M., Ohashi, T., Kobayashi, M., Eto, Y., Miyamura, N., Nishida, K. et al. Identification of Fabry's disease by the screening of alpha-galactosidase $\mathrm{A}$ activity in male and female hemodialysis patients. Clin. Nephrol. 64, 281-287 (2005).

14 Ichinose, M., Nakayama, M., Ohashi, T., Utsunomiya, Y., Kobayashi, M. \& Eto, Y. Significance of screening for Fabry disease among male dialysis patients. Clin. Exp. Nephrol. 9, 228-232 (2005).

15 Rolfs, A., Bottcher, T., Zschiesche, M., Morris, P., Winchester, B., Bauer, P. et al. Prevalence of Fabry disease in patients with cryptogenic stroke: a prospective study. Lancet 366, 1794-1796 (2005).

16 Ishii, S., Suzuki, Y. \& Fan, J. Q. Role of Ser-65 in the activity of alpha-galactosidase A: characterization of a point mutation (S65T) detected in a patient with Fabry disease. Arch. Biochem. Biophys. 377, 228-233 (2000).

17 Ishii, S., Chang, H. H., Kawasaki, K., Yasuda, K., Wu, H. L., Garman, S. C. et al. Mutant alpha-galactosidase A enzymes identified in Fabry disease patients with residual enzyme activity: biochemical characterization and restoration of normal intracellular processing by 1-deoxygalactonojirimycin. Biochem J. 406, 285-295 (2007).

18 Kase, R., Bierfreund, U., Klein, A., Kolter, T., Utsumi, K., Itoha, K. et al. Characterization of two alpha-galactosidase mutants (Q279E and R301Q) found in an atypical variant of Fabry disease. Biochim. Biophys. Acta. 1501, 227-235 (2000).

19 Park, J. Y., Kim, G. H., Kim, S. S., Ko, J. M., Lee, J. J. \& Yoo, H. W. Effects of a chemical chaperone on genetic mutations in alpha-galactosidase $A$ in Korean patients with Fabry disease. Exp. Mol. Med. 41, 1-7 (2009).

20 Okumiya, T., Ishii, S., Takenaka, T., Kase, R., Kamei, S., Sakuraba, H. et al. Galactose stabilizes various missense mutants of alpha-galactosidase in Fabry disease. Biochem. Biophys. Res. Commun. 214, 1219-1224 (1995).

21 Choi, J. H., Cho, Y. M., Suh, K. S., Yoon, H. R., Kim, G. H., Kim, S. S et al Short-term efficacy of enzyme replacement therapy in Korean patients with Fabry disease. J. Korean Med. Sci. 23, 243-250 (2008).

22 Lee, J. K., Kim, G. H., Kim, J. S., Kim, K. K., Lee, M. C. \& Yoo, H. W. Identification of four novel mutations in five unrelated Korean families with Fabry disease. Clin. Genet. 58, 228-233 (2000)

23 Kayganich, K. A. \& Murphy, R. C. Fast atom bombardment tandem mass spectrometric identification of diacyl, alkylacyl, and alk-1-enylacyl molecular species of glycerophosphoethanolamine in human polymorphonuclear leukocytes. Anal. Chem. 64, 2965-2971 (1992).

24 Bligh, E. G. \& Dyer, W. J. A rapid method of total lipid extraction and purification. Can. J. Biochem. Physiol. 37, 911-917 (1959).

25 Desnick, R. J., Allen, K. Y., Desnick, S. J., Raman, M. K., Bernlohr, R. W. \& Krivit, W. Fabry's disease: enzymatic diagnosis of hemizygotes and heterozygotes. Alphagalactosidase activities in plasma, serum, urine, and leukocytes. J. Lab. Clin. Med. 81, 157-171 (1973).

26 Schafer, E., Baron, K., Widmer, U., Deegan, P., Neumann, H. P., Sunder-Plassmann, G. et al. Thirty-four novel mutations of the GLA gene in 121 patients with Fabry disease. Hum. Mutat. 25, 412 (2005).

27 Sachdev, B., Takenaka, T., Teraguchi, H., Tei, C., Lee, P., McKenna, W. J. et al. Prevalence of Anderson-Fabry disease in male patients with late onset hypertrophic cardiomyopathy. Circulation 105, 1407-1411 (2002).

28 Okuda, S. Renal involvement in Fabry's disease. Intern. Med. 39, 601-602 (2000)

29 Ries, M., Gupta, S., Moore, D. F., Sachdev, V., Quirk, J. M., Murray, G. J. et al. Pediatric Fabry disease. Pediatrics 115, e344-e355 (2005).

30 Ries, M., Ramaswami, U., Parini, R., Lindblad, B., Whybra, C., Willers, I. et al. The early clinical phenotype of Fabry disease: a study on 35 European children and adolescents. Eur. J. Pediatr. 162, 767-772 (2003).

31 Garman, S. C. Structure-function relationships in alpha-galactosidase A. Acta. Paediatr. Suppl. 96, 6-16 (2007).

32 Garman, S. C. \& Garboczi, D. N. The molecular defect leading to Fabry disease: structure of human alpha-galactosidase. J. Mol. Biol. 337, 319-335 (2004).

33 Yasuda, M., Shabbeer, J., Benson, S. D., Maire, I., Burnett, R. M. \& Desnick, R. J. Fabry disease: characterization of alpha-galactosidase $A$ double mutations and the D313Y plasma enzyme pseudodeficiency allele. Hum. Mutat. 22, 486-492 (2003).

34 Ledvinova, J., Poupetova, H., Hanackova, A., Pisacka, M. \& Elleder, M. Blood group B glycosphingolipids in alpha-galactosidase deficiency (Fabry disease): influence of secretor status. Biochim. Biophys. Acta. 1345, 180-187 (1997). 